\title{
A EXPANSÃO DA HANSENÍASE NO NORDESTE BRASILEIRO *
}

\author{
Maria de Fátima P. Militåo de Albuquerque** \\ Heloísa Maria Mendonca de Morais*** \\ Ricardo Ximenes****
}

\begin{abstract}
ALBUQUERQUE, M. de F. P. M. de et al. A expansão da hanseníase no nordeste brasileiro. Rev. Saúde públ., S. Paulo, 23:107-116, 1989.

RESUMO: Caracterizou-se a situação epidemiológica da hanseníase na cidade de Recife, Estado de Pernambuco, Brasil, entre 1960 e 1985, pela análise de 3.923 fichas clínico-epidemiológicas de pacientes notificados à Secretaria de Saúde do Estado de Pernambuco, Brasil. Foram calculados os coeficientes de detecção de casos brutos e específicos por sexo, grupo etário e forma clínica, além de analisados o modo de detecção dos casos e o intervalo de tempo decorrido entre o aparecimento dos sintomas e o diagnóstico de hanseníase. O estudo da tendência temporal do coeficiente de detecção de casos revelou um crescimento progressivo de 5,5:100 000 habitantes em 1960 para 36,1:100 000 habitantes, em 1985. O predomínio da forma tuberculóide e o elevado percentual de menores de 15 anos acometidos pela doença podem estar refletindo a expansão da endemia na cidade do Recife, PE. A diminuição e estabilização do intervalo de tempo decorrido desde o aparecimento dos sintomas até o diagnóstico de hanseníase, a partir de 1979, foram consideradas indicadores da detecção mais precoce dos casos e, conseqüentemente, da aproximação do coeficiente de deteç̧ão de casos do coeficiente de incidência. Entre 1970 e 1985, o.modo de detecção de casos mais freqüente foi a consulta dermatológica, seguida pela notificação; apenas $14,2 \%$ dos casos foram descobertos através da vigilância de comunicantes. A análise dos indicadores epidemiológicos e operacionais sugere que 0 aumento expressivo do coeficiente de deteç̧ăo de casos deve ser resultado tanto da expansão da endemia quanto da implementação de algumas das açōes de controle. Já o coeficiente de prevalência calculado para a cidade do Recife, em dezembro de 1985, foi de 2,04/mil habitantes, situando-se a cidade como área de alta endemicidade para a hanseníase, pelos critérios da Organização Mundial de Saúde.
\end{abstract}

DESCRITORES: Hanseníase, incidência. Urbanização, tendências. Prevalência.

\section{INTRODUÇÃO}

A urbanização da hanseníase, como de resto de muitas outras doenças infecciosas e parasitárias, é fato epidemiológico inquestionável, e que vem ocorrendo em todas as áreas endêmicas, inclusive no Brasil 3, 4, 14, 25. Entre nós, segundo alguns trabalhos 8,14 , o fenômeno parece estar particularmente associado aos movimentos migratórios e ao cá́tico assentamento dos migrantes nos espaços urbanos periféricos. Via de regra, a elevada densidade demográfica destes espaços, acrescida do baixo padrão sanitário de sua população, cujo arqui-conhecido diagnóstico seria redundante mencionar aqui, facilitam a difusão do Mycobacterium leprae, um microorganismo de considerável infecciosidade $5,17,38,39,54$.
Mercê de todo este conhecimento, a Região Nordeste do Brasil, com suas grandes áreas urbanas, era tida, até muito recentemente, como "imune" a esta reorganização espacial da doença. Tomada em seu conjunto, ainda continua sendo considerada como a região de mais baixa prevalência no país ${ }^{18,29,33}$.

Contudo, a reunião de avaliação do Programa de Controle da Hanseníase no Brasil, realizada em 1985 pelo Ministério da Saúde, permitiu não somente detectar uma tendência à expansão da endemia no país $29,30,32,33$, como também constatar ser o Nordeste a região que vem apresentando nos últimos anos as maiores taxas de crescimento anual ${ }^{33}$. Este fato, além de fazer ressurgir a velha discussão a respeito das causas de uma pretensa escassez de casos na região("determi-

* Financiado pela Secretaria de Ciência e Tecnologia do Ministério da Saúde. Processo $n^{2} 12744 / 85$

* Departamento de Medicina Clínica da Universidade Federal de Pernambuco - Av. Moraes Rego, s/nº - Cidade Universitária - 50000 - Recife, PE - Brasil.

** Departamento de Medicina Social da Universidade Federal de Pernambuco - CCS - Av. Moraes Rego, $s / n^{2}$. Cidade Universitária - 50000 - Recife, PE - Brasil.

*** Departamento de Medicina Tropical da Universidade Federal de Pernambuco - Av. Moraes Rego, $s / n^{\circledR}-C_{-}$ dade Universitária - 50000 - Recife, PE - Brasil. 
nação genética diferenciada 18 ", clima seco não favorável à disseminação do Mycobacterium leprae 41", "importante sub-registro de casos ${ }^{18 ")}$ veio reacender antigas dúvidas de alguns daqueles que vinham se debruçando sobre a questão. Assim é que as causas genética e climática arroladas, insuficientemente pesquisadas enquanto fatores explicativos de uma desigualdade regional da doença, foram cedendo lugar às evidências que a mera especulação já nảo podia conter.

$O$ baixo índice de prevalência da hanseníase registrado para o Nordeste pode, na realidade, ser decorrente de sub-registro de casos e não estar expressando o nível real de endemicidade na região.

Além disto, os casos notificados para o Estado de Pernambuco vinham mostrando uma predominância da forma tuberculóide e uma freqüência acentuada da doença em menores de 15 anos, ambos indícios de expansão da endemia 29,30.

$\mathrm{Na}$ tentativa, portanto, de elucidar estas e outras questōes na cidade do Recife é que foi realizada a presente investigação, dirigida ao enfoque epidemiológico e do controle da hanseniase nesta grande área urbana do Nordeste Brasileiro.

\section{METODOLOGIA}

Foram analisadas 3.923 fichas clínicoepidemiológicas de casos com diagnóstico definitivo de hanseníase, notificados à Secretaria de Saúde do Estado, entre 1960 e 1985, considerandose apenas os dados relativos aos residentes em Recife, Estado de Pernambuco (PE).

A partir dos dados coletados, foram calculados "coeficientes anuais de deteç̧ão de casos"*. Com base nos coeficientes de detecção brutos e específicos, segundo forma clínica, sexo e grupo etário, realizou-se o estudo da tendência temporal de endemia para o período analisado. Os dados obtidos foram ajustados a uma curva exponencial. Foi realizado o teste de Cox Stuart ${ }^{9}$ para a análise da tendência temporal das séries de coeficientes de deteç̧ão brutos e específicos. Para averiguar as diferenças entre essas séries por sexo, forma clínica e grupo etário, empregou-se o teste de Friedman ${ }^{9}$. Foi estudada ainda a distribuição percentual dos casos por grupos etários e formas clínicas. Para a observação da distribuição dos casos, por grupos etários, efetuou-se a análise de variância e o teste das comparações múltiplas dois a dois de Tukey ${ }^{34}$. Para comparação entre os valores obtidos para cada forma clínica realizouse o teste das comparaçð̄es múltiplas dois a dois.

A média de idade dos doentes ao ser efe- tuado o diagnóstico, em geral e discriminado por formas clínicas, por ano de registro, foi calculada através do método đe distribuição de frequência, por grupos etários, a cada ano. Para a análise da tendência temporal da média de idade de início da doença realizou-se o teste de Cox Stuart ${ }^{9}$.

Analisaram-se ainda os elementos utilizados para a classificação dos 3.923 casos por formas clínicas. Empregou-se o teste de Cox Stuart ${ }^{9}$ para a verificação da tendência na utilização do exame histologico.

O coeficiente de prevalência para o Recife foi calculado com base no número total de pacientes em registro ativo, em 31 de dezembro de 1985. Essa informação foi obtida diretamente, nas diversas unidades de saúde das cidades envolvidas no Programa de Controle.

Além desses indicadores epidemiológicos, foram analisados alguns dos denominados indicadores operacionais, recomendados pela Organização Mundial de Saúde, para avaliação das ações de controle 20,39 . Analisou-se a variação do intervalo de tempo mediano decorrido desde o início dos sintomas até o diagnóstico da doença, para todos os pacientes, a cada ano. A informação foi conseguida a partir das fichas de $90, .5 \%$ dos 3.923 casos revisados. $O$ intervalo de tempo foi estabelecido em meses e para medir a dispersão dos valores em torno da mediana calculou-se a amplitude semi-interquartílica para cada ano. Foi analisado também o modo de detecção dos casos que refletem a intensidade das atividades de vigilância epidemiológica, efetuadas pelo Programa de Controle. Para esta análise foi considerado apenas o período compreendido entre 1970 e 1985, pelo fato de esta informação não aparecer de forma sistemática nas fichas da década anterior.

Os dados demográficos foram fornecidos pela Secretaria de Saúde do Estado de Pernambuco e estimados pelo método geométrico a partir dos censos de 1960, 1970 e 1980. A estrutura da população por sexo e grupo etário está de acordo com os percentuais observados nos referidos censos.

O nível de significância crítico admitido para rejeição da hipótese nula e adotada em todo o tratamento estatístico foi de uma probabilidade máxima de erro de $5 \%(p<0,05)$.

\section{ANÁLISE E DISCUSSÃO DOS RESULTADOS}

O estudo de tendência temporal da hanseníase, no Recife, evidenciou crescimento sigficativo do coeficiente de detecção de casos que passou de 5,5:100 000 habitantes, em 1960, para $36,1: 100000$ habitantes, em 1985. (Fig. 1)

\footnotetext{
* Denominação recomendada por alguns autores 20,21,22,30,39, em substituição a "coeficientes de incidência" por ser mais adequado para dados editados através de atividades de rotina de serviços de saúde.
} 


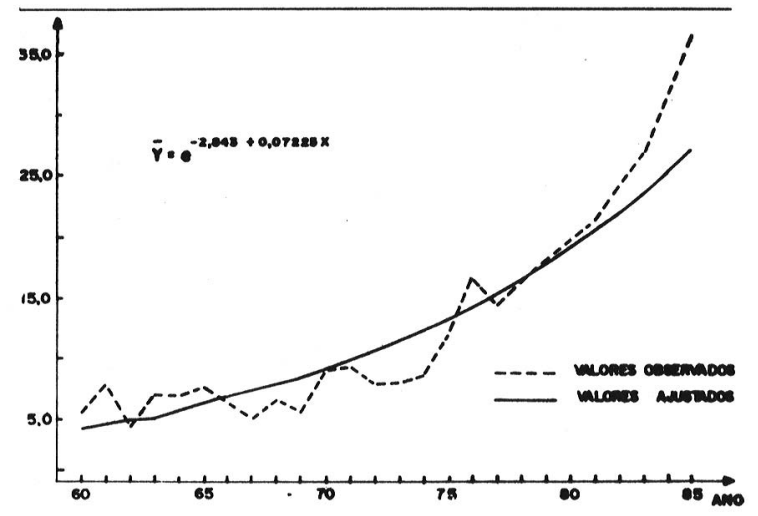

Fig. 1 - Tendência do coeficiente de detecção de casos de hanseniase, valores observados e ajustados. Redfe, 1960 a 1985.

Este aumento poderia ser decorrente da expansão da endemia ou de uma intensificação das atividades do Programa de Controle. Outra alternativa é que os fatores mencionados estariam atuando conjuntamente, o que pode ser demonstrado pela análise dos indicadores epidemiológicos e operacionais, conforme segue.

A análise de tendência do coeficiente de detecção específico por sexo evidenciou que não houve diferença estatisticamente significativa, apresentando ambas as séries tendência temporal crescente. (Tabela 1). Na literatura é comum o relato de maior freqüência de casos entre os homens e, atualmente, vários autores $2,4,10,11,13,19,23$ admitem que o risco de exposição seria o fator responsável pela diferença detectada. Ao considerar os resultados obtidos no presente trabalho, - padrão similar de ocorrência da hanseníase, em ambos os sexos, deve estar expressando, também, oportunidade de exposição semelhante. Este fato poderia ser explicado diante de uma maior mobilidade social das mulheres, principalmente devido à sua inserção progressiva no mercado de trabalho, nos últimos anos, no Brasil ${ }^{24}$.

Ao analisar a distribuição dos casos novos de hanseniase, por grupos etários, verifica-se que $23,1 \%$, o maior percentual de casos, situa-se no grupo entre 15 e 24 anos. O grupo etário de 0 a 14 anos apresentou um percentual de $21,9 \%$, enquanto que para os indivíduos com idade igual ou acima de 60 anos este percentual foi de $10 \%$.

\section{TABELA 1}

Tendência do coeficiente de detecção de casos de hanseníase por cem mil habitantes por sexo. Recife, 1960 a 1985

\begin{tabular}{lrr}
\hline & & Sexo \\
Anos & Masc. & Fem. \\
\hline 1960 & 5,7 & 5,3 \\
1961 & 10,7 & 5,6 \\
1962 & 5,8 & 3,4 \\
1963 & 6,5 & 7,9 \\
1964 & 9,8 & 4,6 \\
1965 & 8,7 & 6,9 \\
1966 & 6,7 & 6,1 \\
1967 & 4,8 & 5,5 \\
1968 & 7,2 & 6,1 \\
1969 & 5,8 & 5,6 \\
1970 & 7,2 & 10,7 \\
1977 & 10,0 & 8,4 \\
1972 & 8,4 & 7,3 \\
1973 & 9,6 & 6,7 \\
1974 & 7,8 & 9,3 \\
1975 & 11,0 & 12,5 \\
1976 & 16,5 & 16,7 \\
1977 & 13,2 & 15,3 \\
1978 & 14,8 & 17,3 \\
1979 & 17,6 & 18,5 \\
1980 & 20,1 & 19,1 \\
1981 & 22,6 & 19,6 \\
1982 & 24,4 & 24,1 \\
1983 & 29,2 & 24,4 \\
1984 & 31,4 & 31,1 \\
1985 & 33,8 & 38,1 \\
\hline
\end{tabular}

A média de idade dos pacientes, no momento do diagnóstico, a cada ano, manteve-se parcialmente constante tanto em relação ao total de casos como para as formas clínicas virchowiana, indeterminada e tuberculóide, ao longo dos 26 anos estudados. (Fig. 2)

O padrão de distribuição da hanseníase, por faixa etária, registrado na literatura é variável e apresenta diferenças regionais marcantes ${ }^{13}$. Em áreas endêmicas, o coeficiente de detecção de casos é maior na faixa etária entre 10 e 20 anos $11,13,42$. Irgens ${ }^{19}$ encontrou uma relação inversa entre a média de idade de início da doença para todos os pacientes a cada ano e o nível de endemicidade. Esta associação poderia ser explicada, segundo o autor, por uma exposição precoce de crianças à infeç̧ão intradomiciliar, em condiçôes de alta morbidade. A propósito, Nossitou e $\mathrm{col}^{36}$ consideram que a proporção de crianças acometidas em regiões onde a doença é altamente preva- 
lente pode chegar a 20\%. Portanto, o aparecimento de casos novos de hanseníase em crianças tem significado epidemiológico importante porque indica a precocidade da exposição e a persistência da transmissão da doença, constituindo-se, assim, indicador do nível endêmico 21,29,31,36

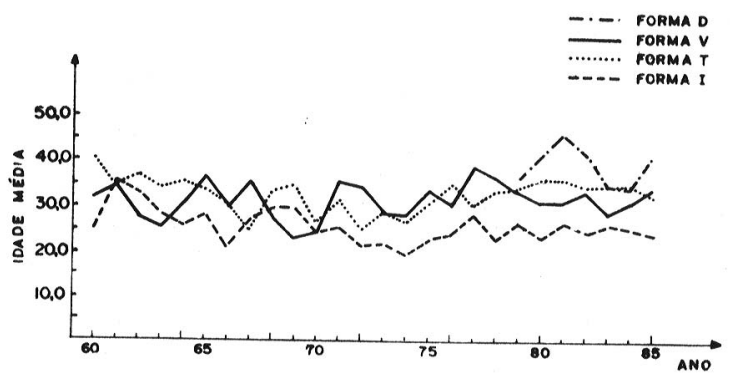

Fig. 2 - Idade média dos pacientes no momento do diagnóstico de hanseniase por forma clínica. Recife, 1960 a 1985.

No Recife, para os grupos etários de 0 a 14 anos e com idade igual ou maior de 15 anos, o coeficiente de detecção de casos apresentou tendência crescente ao longo do período observado. (Tabela 2)

\section{TABELA 2}

Tendência do coeficiente de detecção de casos de hanseníase por cem mil habitantes, por grupo etários. Recife, 1960 a 1985

\begin{tabular}{ccc}
\hline \multirow{2}{*}{ Anos } & \multicolumn{2}{c}{ Grupos Etários } \\
& 0 a 14 anos. & $\geq 15$ anos \\
\hline 1960 & 1,9 & 7,8 \\
1961 & 2,2 & 11,7 \\
1962 & 0,6 & 7,1 \\
1963 & 3,1 & 9,9 \\
1964 & 4,1 & 8,8 \\
1965 & 3,2 & 10,7 \\
1966 & 3,0 & 7,2 \\
1967 & 3,9 & 6,0 \\
1968 & 4,0 & 8,3 \\
1969 & 2,5 & 7,7 \\
1970 & 7,1 & 10,3 \\
1971 & 5,9 & 11,4 \\
1972 & 6,7 & 8,5 \\
1973 & 6,9 & 8,7 \\
1974 & 6,6 & 9,9 \\
1975 & 5,4 & 16,0 \\
1976 & 8,6 & 21,9 \\
1977 & 6,3 & 19,6 \\
1978 & 7,7 & 21,6 \\
1979 & 8,7 & 24,2 \\
1980 & 11,8 & 23,8 \\
1981 & 12,9 & 25,4 \\
1982 & 13,0 & 30,0 \\
1983 & 16,5 & 32,1 \\
1984 & 20,3 & 37,1 \\
1985 & 19,4 & 45,2 \\
\hline
\end{tabular}

Analisando a tendência temporal do coeficiente de detecção de casos por faixas etárias, tomando por base intervalos menores do período de tempo estudado, observa-se que houve um incremento desta taxa em todos os grupos considerados, quando comparados o início e o final do período estudado. $O$ aumento deste coeficiente foi mais pronunciado no grupo com idade igual ou acima de 60 anos. O grupo de 0 a 14 anos apresentou o segundo maior aumento da taxa de detecção com tendência ascendente durante todo o período. (Tabela 3)

TABELA 3

Tendência do coeficiente médio de detecção de casos de hanseníase por cem mil habitantes, por grupo etário e perídodo de tempo. Recife, 1960 a 1985

\begin{tabular}{rccccc}
\hline \multirow{2}{*}{ Tempo } & \multicolumn{5}{c}{ Faixa Etária } \\
\cline { 2 - 6 } & $0-14$ & $15-24$ & $25-39$ & $40-59$ & $\geq 60$ \\
\hline 1960 & & & & & \\
64 & 2,4 & 7,2 & 8,7 & 13,8 & 4,9 \\
1965 & & & & & \\
69 & 3,7 & 6,9 & 5,9 & 11,3 & 10,1 \\
1970 & & & & & \\
74 & 6,6 & 7,9 & 8,2 & 12,8 & 9,8 \\
1975 & & & & & \\
79 & 7,4 & 17,3 & 16,8 & 21,6 & 31,1 \\
1980 & & & & & \\
85 & 15,7 & 26,1 & 31,0 & 34,1 & 48,7 \\
\hline
\end{tabular}

Em síntese, o que parece determinar a diferença entre os coeficientes de incidência da doença nos diversos grupos etários é a oportunidade de exposição precoce ou tardia ao Mycobacterium leprae, determinada pelo maior ou menor nível de endemicidade local.

A deteç̧ão de casos de hanseníase, nos diversos grupos, etários, é também determinada pelo grau de eficácia do Programa de Controle. Assim é que, logo após a introdução de novas atividades de detecção ou da implementação das previamente existentes serão detectados os casos "acumulados". O efeito desta acumulação devese refletir na descoberta de casos antigos, que em geral correspondem às pessoas com mais idade 37 , 39. No Recife, os resultados encontrados revelam alto percentual de casos de hanseníase no grupo etário de 0 a 14 anos, ao lado de um incremento considerável do coeficiente de deteç̧ão de casos nesse mesmo grupo, o que pode estar refletindo a expansão da endemia. Contudo, a análise de tendência da média de idade de início da doença indica que esta se manteve parcialmente constante durante todo o período, não mostrando uma relação inversa, esperada, com o provável au- 
mento da endemicidade. Isto poderia ser explicado pelo aumento do coeficiente de detecção entre pessoas com idade igual ou acima de 60 anos, pela ativação do Programa de Controle a partir do qüinqüênio 1975-1979, influenciando o cálculo da média de idade para todos os casos, a cada ano. $O$ aumento da endemicidade poderia não ter alcançado níveis capazes de provocar a diminuição da média de idade de início da doença. Informe da Organização Mundial de Saúde ${ }^{39}$ refere que uma não diminuição para esta média de idade poderia ocorrer em virtude de os grupos mais jovens da população terem sido imunizados com a vacina $B C G$ (Bacilo Calmette Guérin) o que talvez lhes conferisse uma proteção relativa contra a hanseníase.

Quanto às formas clínicas de ocorrência da doença, ao se analisar os coeficientes de deteç̧ão de casos para as formas virchowiana, indeterminada e tubercoloide, vê-se que os mesmos apresentam tendência crescente, estatisticamente significativa, mais pronunciada para a tuberculóide que ascendeu de 0,8/100 000 habitantes, em 1960, para 19,5/100 000 habitantes, em 1985, tendo este aumento prevalecido tanto ao se considerar a população infantil ( 0 a 14 anos) quanto a adulta (maiores de 15 anos). Seguiram-se, por ordem de freqüência, as formas virchowiana, indeterminada e dimorfa. (Fig. 3 e Tabela 4)

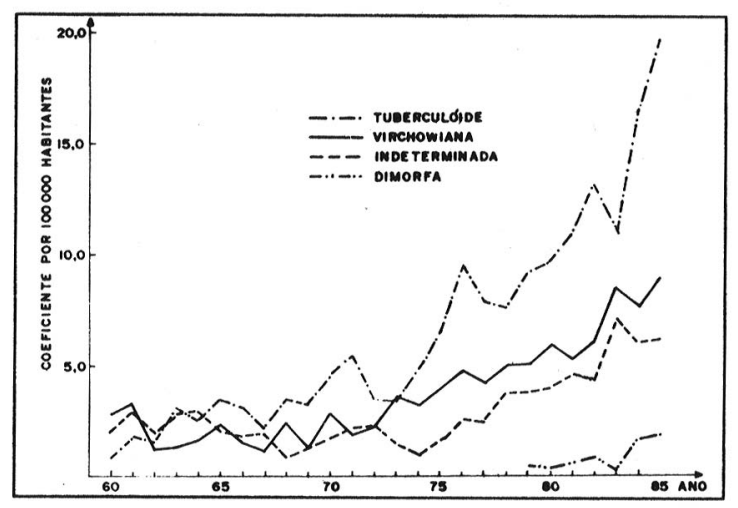

Fig. 3 - Tendência do coeficiente de detecção de hanseniase por forma clínica. Recife, 1960 a 1985.

Ao se considerar o Estado de Pernambuco, o aumento do número de casos de hanseníase, em registro ativo, nos últimos anos, também se fez acompanhar de aumento do percentual da forma turberculóide. No ano de 1985, esta foi a forma que apresentou maior percentual de casos em registro ativo, perfazendo $39,22 \%$, enquanto as formas bacilíferas (virchowiana e dimorfa), conjuntamente, apresentaram $38,72 \%$, e a indeterminada, $22,04 \%$.
Porém, no Brasil, as formas multibacilares têm sido predominantes $6,18,27,28,41$, apesar de um estudo da série histórica da prevalência da endemia no país ${ }^{18}$, no período de 1969 a 1982, haver revelado uma estabilização relativa dos percentuais das formas bacilíferas e da indeterminada e uma "discreta tendência à elevação dos percentuais da forma tuberculóde, ainda não significante estatisticamente".

Em relação aos casos detectados no Recife, parece ser de especial interesse a observação de que a utilização do exame histopatologico para a classificação dos casos aumentou durante o período. Este aumento, estatisticamente significativo a partir de 1974, foi paralelo ao registro de altos percentuais de casos da forma tuberculóide que se mantiveram em torno de $50 \%$. Este fato, por garantir maior acurácia diagnóstica 12,43,44, falaria a favor de predomínio real desta forma clínica da doença na cidade do Recife.

Alguns dados epidemiológicos sugerem não existir correlação entre o número de casos virchowianos e os coeficientes de prevalência total de hanseníase. Alguns autores $1,12,35$ observaram que a proporção de casos virchowianos parece tender a patamar de estabilidade dentro de uma população, ao contrário dos casos tuberculóides que estariam positivamente correlacionados à prevalência global da doença. A correlação entre o nível de endemicidade e o predomínio de casos desta forma clínica está sendo aceita pelo Ministério da Saúde à medida em que adotou, recentemente, a proporção de casos novos da forma tuberculóide como indicador de tendência da endemia ${ }^{31}$. Reconhece-se hoje que a estrutura genética do hospedeiro - por intermédio de fatores ligados ao complexo maior de histocompatibilidade - parece ter influência sobre o tipo de enfermidade que se produz após a infecção através de uma modulação da resposta imuneespecífica ao Mycobacterium leprae $7,15,16,39,52,53$. Sabendo-se que 80 a $90 \%$ da população sadia apresenta uma resposta positiva a Mitsudina ${ }^{1,40}$, torna-se compreensível porque em uma situação de expansão da endemia a forma tuberculóide, que é a expressão de uma resposta imune celular competente 7,16 , torna-se mais freqüente. Assim sendo, o predomínio da forma tuberculóide no Recife poderia estar realmente refletindo a expansão da endemia.

A Secretaria de Saúde registrava, em dezembro de 1985, para todo o Estado de Pernambuco, um coeficiente de prevalência da ordem de 0,7: 1000 habitantes. Enquanto isto, os dados por nós obtidos, na mesma data e a partir de 2.631 casos procedentes da cidade do Recife, correspoderam a um coeficiente de prevalência de 2,04:1 000 habitantes. Nessas condições e de acordo com os crité- 
TABELA 4

Tendência do coeficiente de detecção de casos de hanseníase por cem mil habitantes, por grupo etário e forma clínica. Recife, 1960 a 1985

\begin{tabular}{|c|c|c|c|c|c|c|c|c|}
\hline \multirow[b]{2}{*}{ Anos } & \multicolumn{4}{|c|}{$0-14$} & \multicolumn{4}{|c|}{$\geq 15$} \\
\hline & $\mathrm{V}$ & $\mathrm{D}$ & I & $T$ & $\mathrm{~V}$ & $\mathrm{D}$ & I & $T$ \\
\hline 1960 & 1,0 & - & 10 & - & 29 & - & 27 & 12 \\
\hline 1961 & 0,3 & - & 0,9 & 0,9 & 52 & - & 42 & 24 \\
\hline 1962 & 0,3 & - & 0,3 & - & 1,7 & - & 29 & 2,5 \\
\hline 1963 & 1,1 & - & 0,9 & 1,1 & 1,8 & - & 4,0 & 44 \\
\hline 1964 & 0,8 & - & 2,2 & 1,1 & 2,1 & - & 3,4 & 34 \\
\hline 1965 & 08 & - & 0,8 & 1,6 & 3,2 & - & 2,7 & 4,8 \\
\hline 1966 & 0,8 & - & 20 & 2,0 & 18 & - & 1,6 & 3,9 \\
\hline 1967 & 0,5 & - & 0,7 & 2,7 & 13 & 0,2 & 2,7 & 1,7 \\
\hline 1968 & 1,9 & - & 0,5 & 1,6 & 2,6 & - & 1,1 & 4,5 \\
\hline 1969 & 1,1 & - & 0,5 & 0,9 & 13 & - & 1,5 & 44 \\
\hline 1970 & 2,4 & - & $\tilde{1,}$ & 29 & 3,0 & 0,2 & 1,6 & 5,3 \\
\hline 1971 & 0,5 & - & $1 A$ & 4,0 & 2,6 & - & 2,5 & 6,2 \\
\hline 1972 & 0,9 & - & 28 & 3,0 & 2,9 & 0,2 & 18 & 3,5 \\
\hline 1973 & 2,5 & - & 1,6 & 2,7 & 4,1 & - & 1,1 & 3,6 \\
\hline 1974 & 1,6 & - & 1,1 & 38 & 3,4 & - & 0,7 & 4,5 \\
\hline 1975 & 1,1 & - & 0,9 & 3,4 & 4,4 & - & 1,6 & 62 \\
\hline 1976 & 2,2 & - & 2,0 & 4,4 & 5,9 & - & 2,9 & 12,4 \\
\hline 1977 & 0,9 & - & 1,3 & 4,1 & 6,3 & - & 2,7 & 10,0 \\
\hline 1978 & 1,1 & - & 2,6 & 4,0 & 72 & - & 4,1 & 9,3 \\
\hline 1979 & 1,1 & - & 1,7 & 5,9 & 72 & 0,7 & 4,9 & 10,7 \\
\hline 1980 & $2 A$ & - & 42 & 5,2 & $7 \overline{3}$ & 0,4 & 3,7 & 12,3 \\
\hline 1981 & 3,5 & 0,2 & 3,0 & 6,1 & 5,5 & 0,6 & 4,7 & 12,7 \\
\hline 1982 & $1 A$ & 0,2 & 3,5 & 7,9 & 8,4 & 1,1 & $4 / 4$ & 1,8 \\
\hline 1983 & 5,3 & 02 & 50 & 6,0 & 9,5 & 0,2 & 7,9 & 13,6 \\
\hline 1984 & 48 & 0,9 & 5,5 & 9,1 & 9,4 & 2,0 & 5,9 & 19,7 \\
\hline 1985 & 32 & 0,4 & 4,7 & 11,1 & 114 & 2,6 & 66 & 23,9 \\
\hline
\end{tabular}

rios da Organização Mundial da Saúde, Pernambuco estaria situado em um patamar de endemicidade média e sua capital em níveis de alta endemicidade.

Em anos recentes, o estudo da epidemiologia da hanseníase vem mostrando que a correta interpretação dos resultados obtidos a partir de uma análise dos atributos dos casos detectados indicadores epidmiologicos . necessita de informações relacionadas às atividades de detecção desses casos. Assim é que alguns epidemiologistas terminaram por desenvolver 0 conceito de indicadores operacionais 20,21 .

Nesse sentido, o intervalo de tempo decorrido entre o aparecimento dos sintomas e o diagnóstico da doença é um dado importante que pode ser utilizado como indicador da eficácia operacional do Programa de Controle 20,22. Ele permite avaliar se o coeficiente de detecção está refletindo a incidência real e,em decorrência, termina por fornecer indicações quanto à magnitude do sub-registro. No Recife, o intervalo de tempo decorrido entre o aparecimento dos sintomas e o diagnóstico da doença, referentes a todos os casos novos detectados a cada ano, diminuiu e estabilizou-se em 12 meses a partir de 1979 até o final do período estudado, o que representa uma maior precocidade na detecção de casos a partir do final da década de setenta. (Tabela 5). Também o modo como os casos são detectados é considerado um indicador das atividades de controle. $\mathrm{Na}$ presente investigação, entre 1970 e 1985 , o modo mais freqüente de descoberta de casos novos foi a consulta dermatologica, em $39,7 \%$ dos casos, seguido de perto pela notificação, em $34,9 \%$ dos casos. A vigilância de comunicantes e o reexame de contatos foram responsáveis por apenas $14,2 \%$ dos casos descobertos. (Tabela 6)

Quando o período foi analisado em duas etapas, na tentativa de detectar alguma modificação nas atividades do Programa de Controle, após a Portaria Ministerial de maio de $1976{ }^{26}$, verificouse que não ocorreram mudanças significativas em relação à busca ativa dos casos. Quanto à consulta dermatológica e à notificação, permanecem ambas como os métodos mais freqüentes de deteç̧ão nos dois períodos.

A apresentação espontânea mostrou um aumento considerável de seu percentual, do primeiro para o segundo período. 


\section{TABELA 5}

Intervalo de tempo decorrido desde o aparecimento dos sintomas até o diagnóstico de hanseníase. Recife, 1960 a 1985

\begin{tabular}{ccc}
\hline Anos & $\begin{array}{c}\text { Tempo } \\
\text { Mediano } \\
\text { em Meses }\end{array}$ & $\begin{array}{c}\text { Amplitude } \\
\text { Semi- } \\
\text { Interquartilica } \\
\text { (Q) }\end{array}$ \\
\hline 1960 & 24 & 30 \\
1961 & 24 & 18 \\
1962 & 24 & 18 \\
1963 & 24 & 15 \\
1964 & 12 & 6 \\
1965 & 12 & 6 \\
1966 & 15 & 12 \\
1967 & 24 & 12 \\
1968 & 24 & 12 \\
1969 & 12 & 6 \\
1970 & 12 & 12 \\
1971 & 18 & 12 \\
1972 & 12 & 6 \\
1973 & 12 & 12 \\
1974 & 12 & 15 \\
1975 & 12 & 13,8 \\
1976 & 15 & 10,5 \\
1977 & 18 & 14,5 \\
1978 & 24 & 15 \\
1979 & 12 & 9 \\
1980 & 12 & 9 \\
1981 & 12 & 9,3 \\
1982 & 12 & 9 \\
1983 & 12 & 9,5 \\
1984 & 12 & 9 \\
1985 & 12 & 9 \\
& & \\
\hline & & \\
\hline
\end{tabular}

Como se pode depreender, deixa a desejar a busca ativa de casos do Programa de Controle. Ainda assim, o Programa foi reforçado a partir do final da década de setenta, mediante convênio assinado entre a OXFAM-PE ${ }^{*}$ e a Secretaria de Saúde do Estado, objetivando a intensificação da descoberta de novos casos da doença e a estimulação da demanda espontânea às unidades de saúde 45, 51. Só em 1981, o encaminhamento de casos suspeitos para consulta chegou a resultar em 104 novos diagnósticos, o que equivale a $40 \%$ dos casos registrados em Recife, naquele ano. Data também de 1981 a criação do núcleo de MORHAN - Movimento de Reeintegração do Hanseniano - em Pernambuco. Promovendo a divulgação de informações sobre a doença, de certo, vem contribuindo para a procura dos serviços de saúde pela população.

Ainda assim, são muitas as dificuldades encontradas para o controle da hanseníase. A deficiente infra-estrutura da rede de serviços públicos, acarretando uma baixa intensidade na vigilância dos doentes e seus comunicantes, aliada à fragilidade do sistema de informaçōes que concorre para a dissociação entre o planejamento e a execução das ações de controle, são aspectos que consideramos imprescindíveis de serem revistos no desenvolvimento do Programa de Controle.

Concluindo, a análise dos indicadores

TABELA 6

Distribuição dos casos de hanseníase de acordo com o modo de detecção. Recife, 1970 a 1985

\begin{tabular}{|c|c|c|c|c|c|c|}
\hline \multirow{2}{*}{$\begin{array}{l}\text { Modo de } \\
\text { Detecção }\end{array}$} & \multicolumn{2}{|c|}{$1970-77$} & \multicolumn{2}{|c|}{$1978-85$} & \multicolumn{2}{|c|}{$1970-85$} \\
\hline & $\mathrm{N}^{\mathrm{e}}$ & $\%$ & Ne & $\%$ & $\mathrm{~N}^{2}$ & $\%$ \\
\hline \multirow{7}{*}{$\begin{array}{l}\text { Apresentação } \\
\text { espontânea } \\
\text { Consulta } \\
\text { dermatológica } \\
\text { Notificação } \\
\text { ou denúncia } \\
\text { Investigação } \\
\text { epidemiológica } \\
\text { Reexame de } \\
\text { contatos } \\
\text { Exame de } \\
\text { coletividade } \\
\text { Exame de } \\
\text { população } \\
\text { Outros }\end{array}$} & 32 & 4,7 & 198 & 9,6 & 230 & 8,2 \\
\hline & 268 & 39,0 & 844 & 40,9 & 1.112 & 39,7 \\
\hline & 322 & 39,4 & 656 & 31,8 & 978 & 34,9 \\
\hline & 8 & 12 & 108 & 5,2 & 116 & 4,1 \\
\hline & 99 & 14,4 & 183 & 8,9 & 282 & 10,1 \\
\hline & 5 & 0,7 & 2 & 0,1 & 7 & 0,3 \\
\hline & $\begin{array}{l}2 \\
2\end{array}$ & $\begin{array}{l}0,3 \\
0,3\end{array}$ & $\begin{array}{r}4 \\
68\end{array}$ & $\begin{array}{l}0,2 \\
3,3\end{array}$ & $\pi^{6}$ & $\begin{array}{l}0,2 \\
2,5\end{array}$ \\
\hline Total & 738 & 100,0 & 2.063 & 100,0 & 2.801 & 100,0 \\
\hline
\end{tabular}

Lepra the british leprosy relief Association/Oxfam-PE 
epidemiológicos e operacionais para o período estudado sugere que o aumento expressivo do coeficiente de detecção de casos observados, para o período estudado deve ser resultado tanto da expansão da endemia quanto da implementação de algumas das ações de controle.

\begin{abstract}
ALBUQUERQUE, M. de F. P. M. de et al. [The increase of leprosy in the Northeastern region of Brazill. Rev. Saúde públ., S. Paulo, 23:107-116, 1989.

ABSTRACT: The epidemiological aspects of hanseniasis in Recife from 1960 to 1985 were studied. Clinical-epidemiological records of 3,923 leprosy patients reported to the Secretaria de Saúde do Estado de Pernambuco were reviewed. The cruce as well as the age, sex and type-specific detection rates were calculated. The way the cases were detected and the time elapsed between the appearance of the first symptoms and the disease was analysed. The analysis of the time trend during the observation period showed an increase in the detection rate with time, rising from 5.5 per 100,000 inhabitants in 1960 to 36.1 per 100,000 inhabitants in 1985 . The higiner frequency of the tuberculoid type of leprosy and the high percentage of patients under 15 might refleet the expansion of the disease in $R e$ cife. The decline and the stabilization in the time elapsed between the appearance of the disease and its detection, from 1979 onwards, indicates a more prompt detection and, as a consequence of that, that the rate of detection is approaching the incidence rate. From 1970 to 1985 the most common means of detecting cases of Hanseniasis was through dermatological consultation followed by disease notification. Only $14.2 \%$ of the cases were discovered through the surveillance of contacts. The analysis of the epidemiological and operational indicators suggest that the increase in the detection rate over the period from 1960 to 1985 was due both to expansion of the disease and improvement in control measures. The prevalence rate of Hanseniasis in Recife in December 1984 was 2.04 per 100,000 inhabitants; according to the WHO criteria Recife may be considered an area of high endemicity.
\end{abstract}

KEYWORDS: Leprosy, Occurrence. Urbanization, tendency. Prevalence.

\title{
REFERÊNCIAS BIBLIOGRÁFICAS
}

1. BEIGUELMAN, B. Lepra e genética. Cienc. e Cult., 34: $1121-46,1981$.

2. BEIGUELMAN, B. et al. Lepra e sexo. Rev. paul. Med., 72: 120-9, 1968.

3. BELDA, W. Aspectos epidemiológicos da hanseníase no Estado de São Paulo, em 1974. Hansenol. int., 1: 11$23,1976$.

4. BELDA, W. \& LOMBARDI, C. A incidência da hanseníase no Estado de São Paulo, em 1978. Hansenol. int., 4: 98-112, 1979.

5. BJUNE, G. The lymphocyte transformation test in leprosy with special reference to its use in epidemiology. Leprosy Rev., 52: 241-50, 1981.

6. BRITTO, R. S. Hanseníase na região norte. Hiléia méd., 3 (2) : 37-41, 1981.

7. BULLOCK, W. E. Leprosy: a model of immunological perturbation in chronic infection. $J$. infect. Dis., 137: 341-54, 1978.

8. COLONIZAÇÃO do espaço urbano pelos parasitas. RadisSúmula, Rio de Janeiro, 2: 1-2, nov. 1985.

9. CONOVER, W. J. Practical non parametric statistics. New York, John Wiley, 1980.
10. DOULL, J. A. The epidemioly of leprosy present status and problems. Int. J. Leprosy, 30: 48-66, 1962.

11. DOULL, J. A. et al. The incidence of leprosy in Cordova and Talisay, CEBU, P. I. Int. J. Leprosy, 10: 107-31, 1942.

12. FINE, P. E. M. Problems in the collection and analysis of data in leprosy studies. Leprosy Rev., 52: 197-206, 1981.

13 FINE, P. E. M. Leprosy: the epidemiology of a slow bacterium. Epidem. Rev., 4: 161-88, 1982.

14. GANAPATI, R. Urban leprosy control. Trop. Doct., 13: 76-83, 1983.

15. GODAL, T. Immunological aspects of leprosy: present status. Basel, Karger, 1978. p. 211-42. (Progress in Allergy, 25)

16. GODAL, T. Leprosy immunology: some aspects of the role of the immune system in the pathogenesis of disease. Leprosy Rev., 55: 407-14, 1984.

17. GODAL, T. \& NEGASSI, K. Subclinical infection in leprosy. Brit. med. J., 3: 557-9, 1973.

18. GONÇALVES, A. Epidemiologia e controle da hanseníase no Brasil. Brasília, Ministério da Saúde. Divisão Na- 
cional de Dermatologia Sanitária, 1983.

19. IRGENS, L.M. Leprosy in Norway. Leprosy Rev., 51: 1$127,1980$.

20. LECHAT, M.F. et al. An information system for leprosy control (OMSLEP recording and reporting system). Int. J. Leprosy, 48: 51-61, 1979.

21. LECHAT, M.F. et al. OMSLEP - Systéme d'enregistrement et de notification des malades de la lepre. 2ème ed. Bruxelles, Université Catholique de Louvain, 1983.

2 LECHAT, M.F. et al. Analysis of trends in the occurence of leprosy. Wld Hlth Statist. Quart., 39: 129-37, 1986.

23 LOMBARDI, C. Aspectos epidemiol6gicos da mortalidade entre doentes de hanseníase no Estado de São Paulo (1931-1980). Rev. Saúde públ., S. Paulo, 18: 71-107. 1984.

24. LUCCHESI, C.P. Mulher disputa mais o mercado de trabalho. Folha de S. Paulo, 4 out. 1987.

25. MELLO, A. de. Hanseníase nas áreas metropolitanas. Bol Div. nac. Dermat. sanit, 36: 31-40, 1977.

26. MINISTERRIO DA SAÚDE: Instruções para execução das normas de controle da hanseníase, baixadas pela Portaria $n^{2}$ 165/Bsb, de 14 de maio de 1976. Bol. Div. nac. Der. mat. sanit, 36: 7-12, 1977.

27. MINISTERIO DA SAUDE. Tópicos em hansenologia. Brasília, 1982.

28. MINISTÉRIO DA SAÚDE. Proposta de reforço d̀ implementação do controle da hanseníase no Brasil. Brasília, 1983.

29. MINISTÉRIO DA SAUUDE. Relatório de consultoria sobre avaliaçáo do programa de controle de hanseniase no Brasil. Brasîlia, 1985.

30. MINISTÉRIO DA SAUDE. Dados básicos sobre o controle da hanseniase no Brasil, referentes a 1985. Brasilia, 1986.

31. MINISTÉRTO DA SAÚDE. Relatório do Grupo Técnico; instruçöes normativas, regulamentação referente à Portaria Ministerial n²165 de 1976. Brasilia, 1986.

32. MINISTÉRIO DA SAUDE. Secretaria Nacional de Programas Especiais de Saúde. Divisão Nacional de Dermatologia Sanitária. Dados básicos sobre o controle da han. seniase no Brasil, referentes a 1984. Brasília, 1985.

33. MINISTÉRIO DA SAUDE. Secretaria Nacional de Programas Especiais de Saúde. Diretrizes do Programa de Controle da Hanseníase, 1986-1990. Brasília, 1986. [Mimeografado].

34. MONTGOMERY, D.D. Design and analyse of experiments. New York, John Wiley, 1976.

35. NEWELL, K.W. An epidemiologist's view of leprosy. Bul. Org. mond. Santé, 34: 827-57, 1966.

36. NOSSITOU, F.M. et al. Lepre infantil. Ginebra, Organización Mundial de la Salud, 1976.
37. ORGANIZAÇÃO MUNDIAL DA SAÚDE. A Organização Mundial da Saúde e a hanseníase. Recife, OXFAMCERPHA, 1977. (Relatório em Português, 5).

38. ORGANIZACION MUNDIAL DE LA SALUD. Comité de Expertos en Lepra, Ginebra, 1970. Informe 4. Ginebra, 1970. (Série de Informes Técnicos, 459).

39. ORGANIZACION MUNDIAL DE LA SALUD. Grupo de Estudio sobre Epidemiologia de la Lepra en Relación con la Lucha Anti-leprosa, Ginebra, 1983. Informe. Ginebra, 1985. (Serie de Informes Técnicos, 716).

40. ORGANIZAÇÃO PAN-AMERICANA DA SAÚDE. $M a-$ nual para o controle da hanseníase. Washington, D.C. 1983.

41. ORGANLZACIÓN PANAMERICANA DE LA SALUD. La lepra en las Américas. Bol. Epidem., 4(6): 1-7, 1983.

42. RAO, P.S.S. et al. Incidence of leprosy in gudiyatham Taluk, South India. Ind. J. med. Res., 60: 97-105, 1972.

43. RIDLEY, D.S. Histological classification and the immunological spectrum of leprosy. Bull.Wld Hlth Org., 5l: 45165,1974

44. RIDLEY, D.S. \& JOPLING, W.H. Classification of leprosy according to immunity; a five group system. Int. $J$. Leprosy, 34: 255-73, 1966.

45. SECRETARIA DE SAÚDE DE PERNAMBUCO. Fundação de Saúde Amaury de Medeiros. Plano de trabalho para intensificação da descoberta de novos casos de hanseníase no Estado de Pernambuco, integrado Secretaria de Saúde/FUSAM/OXFAM-PE. Recife, 1980.

46. SECRETARIA DE SAÚDE DE PERNAMBUCO. Fundação de Saúde Amaury de Medeiros. Relatório do trabalho de intensificação da descoberta de novos casos de hanseníase, integrado Secretaria de Saúde/FUSAM/ OXFAM-PE. Recife, 1981.

47. SECRETARIA DE SAÚdE DE PERNAMBUCO. Fundação de Saúde Amaury de Medeiros. Coordenação de Epidemiologia. Relatório final do trabalho de intensificação da descoberta de novos casos de hanseníase, integrado Secretaria de Saúde/FUSAM/OXFAM-PE. Recife, 1985.

48. SECRETARIA DE SAÚdE DE PERNAMBUCO. Fundação de Saúde Amaury de Medeiros. Coordenação de Epidemiologia e Planejamento. Plano de Trabalho para intensificação da descoberta de novos casos de hanseníase. Recife, 1978.

49. SECRETARIA DE SAUUDE DE PERNAMBUCO. Fundação de Saúde Amaury de Medeiros. Coordenação de Serviço Social. Relatório das atividades do plano de intensificaçäo de descobertas de novos casos de han. seniase. Recife, 1980.

50. SECRETARIA DE SAÚDE DE PERNAMBUCO. Fundação de Saúde Amaury de Medeiros. Coordenação de Serviço Social. Relatório síntese de trabalho de intensificação da descoberta de novos casos de hanseníase, integrado Secretaria de Saúde/FUSAM/OXFAM-PE. Recife, 1982. 
51. SECRETARIA DE SAÚDE DE PERNAMBUCO. Fundação de Saúde Amaury de Medeiros. Coordenação de Serviço Social. Relatório síntese do trabalho de intensificação da desooberta de novos casos de hanseníase, integrado Secretaria de Saúde do Estado/FUSAM/OXFAMPE. Recife, 1984.

52. ULRICH, M. La función de los linfocitos en la immunologia de la lepra. In: Seminario Panamericano sobre el Control de la Lepra, 4², Caracas, 1975. Washington, D.C., Organización Panamericana de la Salud, 1977. p. 49-51. (OPAS- Publicación Científica, 344).
53. VAN VOORHIS, W.C. et al. The cutaneous infiltrates of leprosy: cellular characteristics and the predominant $T$ cell phenotypes. New Engl. J. Med., 307: 1593-7, 1982.

54. YOUNG, D.B. et al. A spot test for detection of antibodies to phenolic glycolipid I. Leprosy Rev., 56: 193-8, 1985.

Recebido para publicaçāo em 30/6/1988

Reapresentado em 22/2/1989

Aprovado para publicaçáo em 23/2/1989 